\title{
A Mechanical Construction for the Quartic Trisectrix.
}

By H. Poole.

Figure 46.

The model consists of a circular template, of radius $a$, hinged at $O$, a point on its circumference, to a bar $O A$, where $O A=a$. $P$ is the centre of the circle, and $E$ is the middle point of OP. $C$ is a point on the circumference such that $O P=O C=a$, so that CE is perpendicular to $O P$.

From $\mathrm{E}$ two arms $\mathrm{EX}$ and $\mathrm{EY}$ radiate, and are so arranged by a linkage that the angles $\mathrm{YEC}$ and $\mathrm{XEC}$ are equal.

To trisect an angle with the instrument, place $O A$ along one side, make the arm EX fall on $A$, and open or close $A O C$, EX running along $A$, until the point where the arm EY cuts the circumference of the template lies on the other side of the angle. Let this point be called $B$.

Then $\mathrm{OC}$ is one of the trisectors of the angle AOI3.

Since $\mathrm{OP}=\mathrm{OC}=\mathrm{OA}=a$,

$\therefore A, C, P$ are on the circumference of a circle equal to OCB, which cuts it at $C$, and has $O$ for its centre.

Now $C E$ is perpendicular to $O P$, which joins the centres.

$\therefore \operatorname{arcCB}=\operatorname{arcCA}$.

$\therefore$ angles $\mathrm{CPB}$ and $\mathrm{COA}$ are equal.

But angle $\mathrm{CPB}=2$. angle $\mathrm{COB}$,

$\therefore$ angle $\mathrm{COA}=2$. angle $\mathrm{COB}$,

or $O C$ is one of the trisectors of the angle $A O B$.

Figure 47.

To draw the curve, let $O A$ be fixed, and let the arm EX always run along $A$ : then the point $B$ describes the Quartic Trisectrix. 
Let $B$ be any point on the curve.

With centre $O$, and radius $O A$ describe a circle $A C P$.

Join $\mathrm{BA}$, cutting the circle $\mathrm{ACP}$ at $Q$ : join $O Q$.

Then, obviously, $O Q=O A$, and the angles $O Q A$ and $O A Q$ are equal. Also, from the congruence of the triangles $B P E$ and $A O E$, we see that PBAO is a trapezium.

Hence the angles OQA, OAQ, and PBQ are equal.

Hence $O Q$ is equal and parallel to $P B$.

Therefore $\mathrm{QBPO}$ is a parallelogram, and $\mathrm{QB}=\mathrm{OP}$ and is constant.

It follows that the locus of $B$ is that particular kind of Limaçon which is called the Quartic Trisectrix.

\section{Figure 48.}

Another mechanical method of drawing the Iimaçon is given by the linkage shown in the figure.

Here $\mathrm{AB}=\mathrm{OC}=a, \mathrm{OA}=\mathrm{CB}=\mathrm{DE}=2 a, \mathrm{CD}=\mathrm{BE}=4 a$, and, in the case of the trisectrix, DP $=O D$. $O$ and $A$ are fixed, and $P$ describes the curve.

From the arrangement it will be seen that $\angle A O C=\angle C D E$.

$$
\therefore \quad-A O D=\angle C D P \text {. }
$$

Thus $P$ moves so that $O D=D P$ and $\angle A O D=\angle O D P$, thus describing the trisectrix.

Other Limaçons may also be drawn, the eccentricity depending on the length of DP. 